\title{
Infestation spontanée d'un Dermaptère par des larves de Pseudophysaloptera vincenti n.sp., parasite du Lémurien Galagoides demidovii \\ (Fischer, 1808)
}

\author{
par J.-C. QUENTIN
}

Station expérimentale de La Maboké (République Centrafricaine)

Laboratoire de Zoologie (Vers) associé au C.N.R.S. ( $\mathrm{P}^{r}$ A.-G. Chabaud)

Muséum National d'Histoire Naturelle - 57, rue Cuvier - F 75 -Paris (5)

\begin{abstract}
Résumé
Description de l'espèce, Pseudophysaloptera vincenti n. $\mathrm{sp}$. parasite du Lémurien Galagoides demidovii (Fischer, 1808) en République Centrafricaine. Des larves infestant spontanément l'Insecte Labidura riparia dans la même localité et dont l'adulte est obtenu expérimentalement chez un Rongeur sont identifiées à cette espèce.
\end{abstract}

\begin{abstract}
The encapsulated Nematodes collected in a Forficula from Africa : Labidura riparia Pallas has been identified as third stage larvae of Pseudophysaloptera vincenti $\mathrm{n}$. sp. The adult is a parasite from Africa Lemurid: Galagoides demidovii (Fischer, 1808).
\end{abstract}

\section{Introduction.}

Au cours de recherches de stades larvaires d'Helminthes chez des Insectes collectés à la station Expérimentale de La Maboké en juillet et août 1968, deux larves d'un Nématode Spiruride ont été recueillies chez un Dermaptère Labidura riparia.

Une de ces larves donnée par voie buccale à un Rongeur né en laboratoire a permis d'obtenir un adulte mâle de Physalopteridae. 
Les recherches dans la faune locale ont conduit à comparer ce mâle à ceux de Pseudophysaloptères, récoltés respectivement chez un Lémurien Galagoides demidovii et chez une Musaraigne Crocidura sp.

\section{Description.}

\section{1 - LARVES PARASITES DE Labidura riparia.}

Ces larves au nombre de deux, sont légèrement mobiles à l'intérieur d'une mince capsule dilatant la paroi du côlon de l'Insecte. Libérées par dissection (fig. 1 A) elles présentent les caractères des larves du $3^{\circ}$ stade de Physalopteridae:

- La tête séparée du corps par un sillon, a une bouche allongée dorso-ventralement, limitée par deux pseudolèvres à bords tribolés (fig. $1 \mathrm{~B}$ ). Les lobes médians et latéraux ne présentent aucune différenciation. Ohaque pseudolèvre porte deux papilles submédianes et une amphide. La cavité buccale est profonde de $17 \mu$ (fig. $1 \mathrm{C}$ ).

— Le corps est trapu, long de $1.380 \mu$, large de $96 \mu$. Son organisation interne est parfaitement différenciée. L'anneau nerveux, les deirides et le pore excréteur sont respectivement situés à 120,150 et $225 \mu$ de l'apex. Le pore excréteur est relié à la cellule excrétrice par un fin canal qui décrit une anse convexe vers l'avant. Les différentes régions du tube digestif sont nettement délimitées. Les parties musculaire et glandulaire de l'œsophage mesurent $120 \mu$ et $470 \mu$. L'ébauche génitale pluricellulaire, longue de $120 \mu$, est située à $875 \mu$ de l'apex. La queue mesure $115 \mu$.

\section{2. - Adulte ơ obtenu expérimentalement.}

Un jeune Thamnomys surdaster (Peters), né en laboratoire, est infesté par voie buccale avec une des deux larves. Le Rongeur, autopsié 43 jours après, présente au niveau des canaux biliaires, un mâle de Physalopteridae. Celui-ci, étudié aussitôt après l'autopsie, montre une extrémité céphalique (fig. $1 \mathrm{~F}, \mathrm{G}, \mathrm{H}$ ) constituée de deux pseudolèvres ornées chacune d'un groupe interne de trois dents et d'une petite dent externolatérale. Un étroit collier cuticulaire sépare la tête du reste du corps. Ce spécimen mâle ne porte pas d'ailes latérales, mais un léger renflement de la cuticule céphalique qui se termine à $275 \mu$ de l'apex.

Ses dimensions sont: longueur $7.950 \mu$, largeur $275 \mu$, anneau nerveux, deirides, pore excréteur respectivement situés à 325,460 et $500 \mu$ de l'apex.

Pharynx profond de $85 \mu$. Régions musculaire et glandulaire de l'œsophage longues de 340 et $1.700 \mu$; longueur de l'intestin : $3.500 \mu$.

Le testicule naît à $450 \mu$ de la tête et remonte jusqu'à $410 \mu$ en arrière de celle-ci. La bourse caudale (fig. 1 D) présente 2 ailes hautes de $700 \mu$, larges de $120 \mu$, réunies dans la région précloacale en un bourrelet vésiculeux haut de $130 \mu$ environ.

Quelques granulations et de rares stries cuticulaires sont visibles juste en avant du cloaque. Les papilles cloacales (fig. 2 D) sont petites. Elles sont au nombre de dix paires, plus une papille impaire précloacale. Sur le côté droit, quatre papilles, parmi 

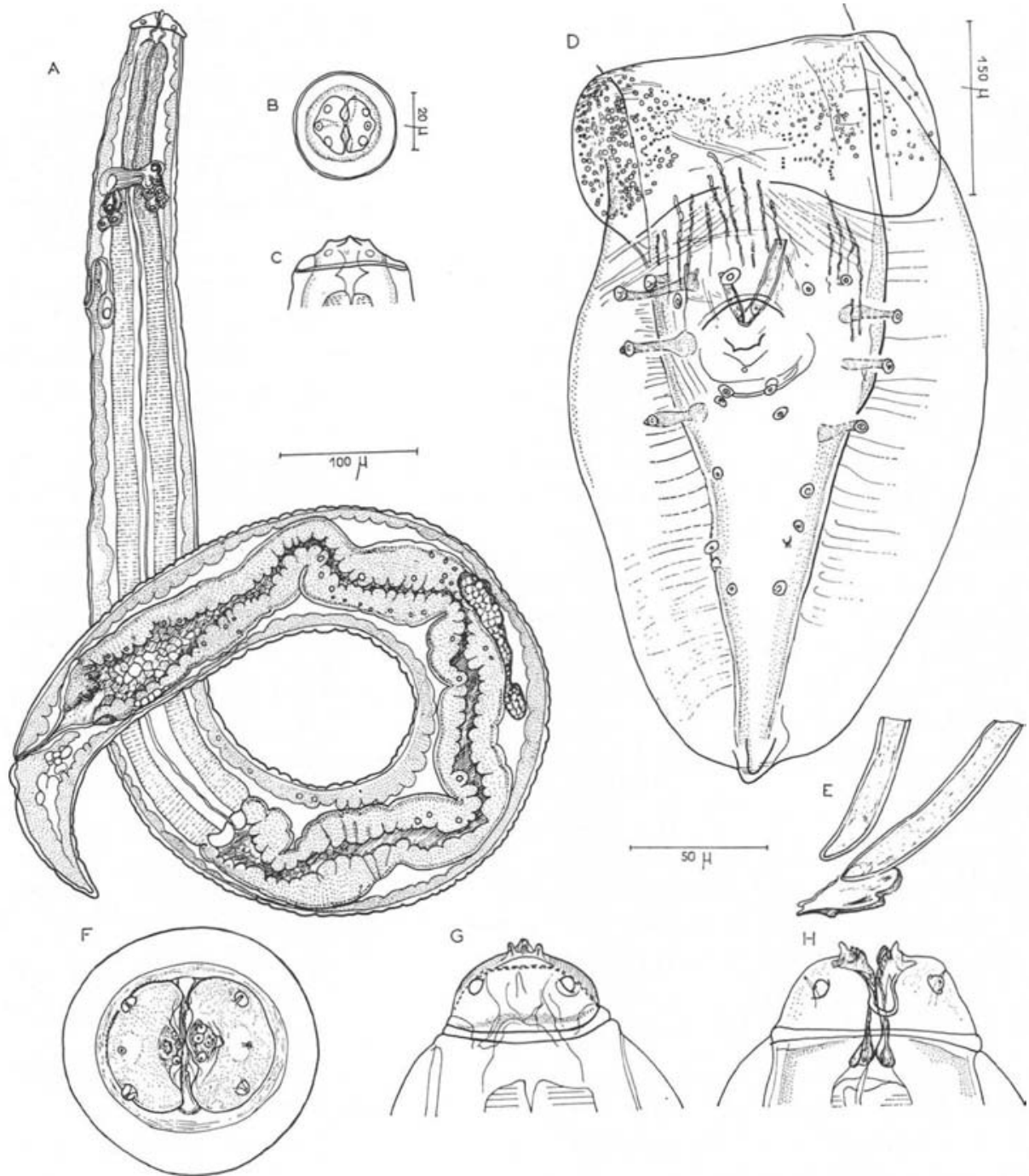

FIG. 1. - Pseudophysaloptera vincenti n. sp. A: larve du $3^{e}$ stade, parasite de Labidura riparia, extraite de sa capsule, vue latérale. B : tête, vue apicale. C: tête, vue latérale. D : bourse caudale, vue ventrale de l'adulte parasite de Thamnomys surdaster. E : détail des spicules et du gubernaculum. F, $\mathrm{G}$ et $\mathrm{H}$ : tête de ce mâle successivement représentée en vues apicale, latérale et ventrale. $\mathrm{A}$ : éch. $100 \mu$. B, C : éch. $20 \mu$. D : éch. $150 \mu$. E, F, G, H : éch. $50 \mu$

les plus latérales, sont pédonculées, trois seulement le sont sur le côté gauche. Les phasmides sont situées entre les deux dernières paires postcloacales. Les spicules, larges de 10 à $11 \mu$ sont inégaux. Le gauche mesure $85 \mu$, le droit $58 \mu$. Le gubernaculum faiblement chitinisé est long de $31 \mu$. La queue mesure $174 \mu$. 
3. - Adultes ơ Parasites de Galagoides demidovii (Fischer, 1808).

Un Lémurien Galagoides demidovii (Fischer, 1808) capturé à la station de La Maboké, en août 1968, est parasité au niveau de l'estomac par deux larves femelles et deux jeunes mâles de Spiruride.

Les mâles (holotypes $\mathrm{n}^{\circ} 220 \mathrm{SA}$ ) mesurent $4.200 \mu$. Ils présentent les caractères céphaliques et génitaux suivants :

- Les papilles céphaliques sont de petite taille, chaque pseudolèvre porte une petite dent externo-latérale (fig. $2 \mathrm{~A}, \mathrm{~B}, \mathrm{C}$ ) et un trident interne peu développé. Le collier cuticulaire qui sépare les lèvres du reste du corps et le renflement cuticulaire qui lui fait suite sont identiques à ceux observés précédemment sur le spécimen obtenu expérimentalement chez Thamnomys surdaster.

- la bourse caudale de chacun des deux mâles (fig. 2 D, E) est ornée de rares lignes cuticulaires précloacales. Il existe dix paires de papilles plus une papille impaire précloacale.

Quatre paires de papilles pédonculées sont visibles sur l'un des mâles. Sur le second, quatre papilles pédonculées sont mises en évidence sur le côté gauche du cloaque, deux seulement le sont sur le côté droit.

Chez les deux mâles, les phasmides sont situées entre les deux dernières paires de papilles post-cloacales.

Les spicules gauche et droit très faiblement chitinisés mesurent respectivement $85 \mu$ et $55 \mu$.

Ces mâles ont par ailleurs les dimensions suivantes: largeur $210 \mu$; anneau nerveux, pore excréteur et deirides situés respectivement à $170,300 \mu$ et $300 \mu$ de l'apex; longueur des portions œsophagienne et glandulaire de l'œsophage $210 \mu$ et $1.060 \mu$; longueur de l'intestin $2.700 \mu$. Queue longue de 200 à $215 \mu$.

\section{4. - Adultes ơ ET $q$, parasites de Crocidura sp.}

Une Crocidura sp. (n ${ }^{\circ} 79$ SA) autopsiée à la station de La Maboké en juillet 1968 présente, à l'intérieur d'une tumeur réactionnelle du duodénum, un mâle et une femélle de Physalopteridae. Ces Nématodes de petite taille $(4 \mathrm{~mm})$ ont les caractères céphaliques et génitaux suivants :

- Chaque pseudolèvre (fig. $2 \mathrm{G}, \mathrm{H}, \mathrm{I}$ ) est ornée de trois dents internes saillantes et d'une dent externo-latérale ; le bord buccal est très légèrement denticulé. Les papilles céphaliques submédianes sont volumineuses.

- La bourse caudale (fig. $2 \mathrm{G}$ ) large et enveloppante ne porte aucune ornementation cuticulaire pré et post-cloacale, mais uniquement deux paires de papilles légèrement pédonculées en avant du cloaque et cinq paires de papilles sessiles en arrière du cloaque.

Nous n'avons pu discerner de spicules. 


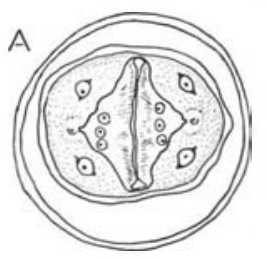

D

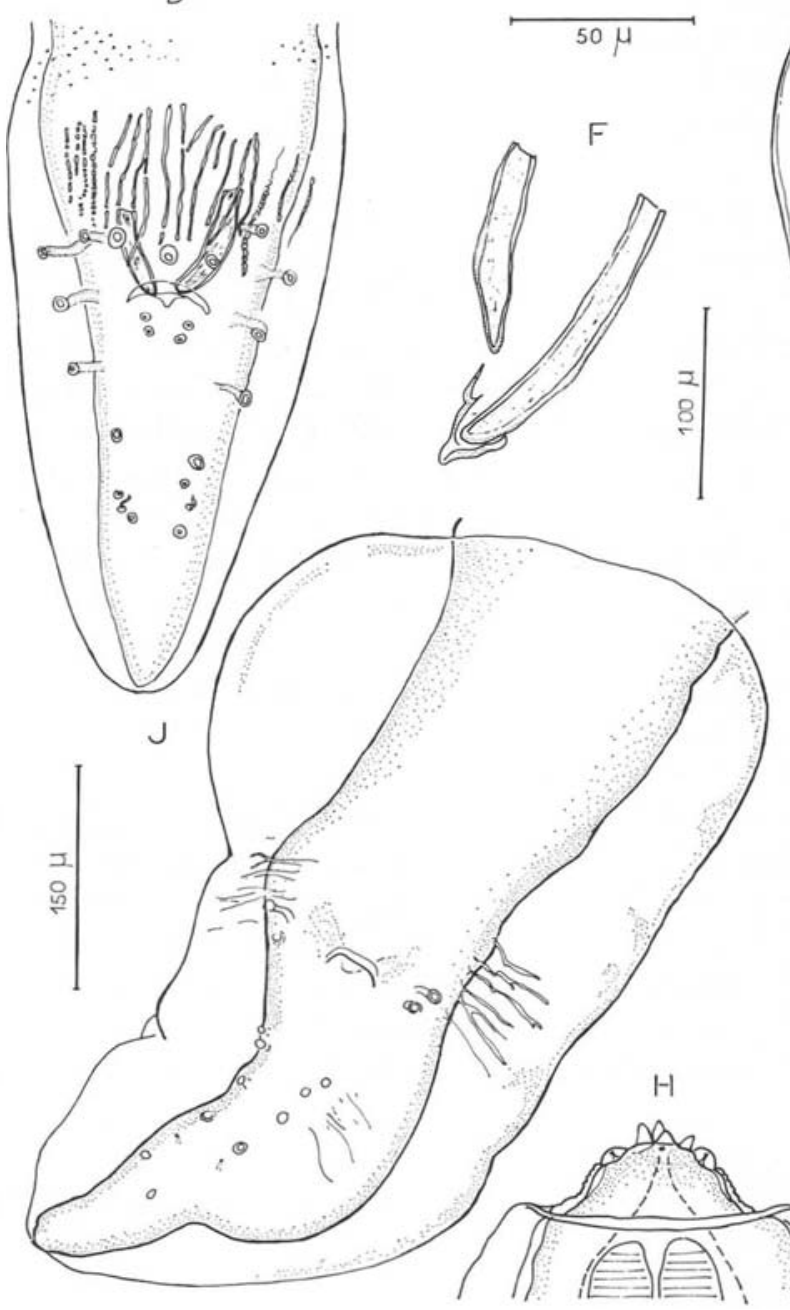

Fig. 2. - Pseudophysaloptera vincenti n. sp. parasite de Galagoides demidovii. A, B et C: tête représentée successivement en vues apicale, latérale et ventrale. D et E : bourses caudales de chacun des deux mâles. F: détail des 2 spicules et du gubernaculum. Pseudophysaloptera soricina, Baylis, 1934. G, H et I : tête représentée en vues apicale, latérale et ventrale. J : bourse caudale du mâle, vue ventrale. A, B, C, F, G, H, I : éch. $50 \mu . \mathrm{D}, \mathrm{E}$ : éch. $100 \mu . \mathrm{J}$ : éch. $150 \mu$ 


\section{Discussion.}

Une infestation spontanée d'un Labidura riparia du Nord de l'Inde par des larves de Physalopteridae a été signalée par Basir en 1948. Cet auteur observe deux larves libres dans la cavité générale de l'Insecte. Elles mesurent chacune 2,1 $\mathrm{mm}$ de long et diffèrent par conséquent de nos spécimens larvaires.

Les différents adultes de Physalopteridae récoltés successivement chez Thamnomys surdaster (Peters) (infestation expérimentale), chez Galagoides demidovil (Fischer, 1808) et Crocidura sp. (infestation spontanée) ont en commun les caractères suivants : - faible taille des mâles et des femelles, — ornementation caudale du mâle presque inexistante, - instabilité des papilles cloacales pédonculées, - longueur réduite et transparence des spicules.

Nous les classons dans le genre Pseudophysaloptera Baylis, 1934.

L'espèce qui, par sa localisation géographique, est la plus proche de nos spécimens est $P$. soricina Baylis, 1934, parasite de Crocidura sp. au Tanganyika.

Nous identifions à cette espèce, le mâle et la femelle que nous avons récoltés chez Crocidura sp. (SA 79) en République Centrafricaine, car i'hôte, sa géographie, la taille des parasites : $4 \mathrm{~mm}$, la morphologie caudale du mâle $\left(^{*}\right)$ sont comparables.

Par ailleurs, les mâles de Pseudophysaloptères infestant expérimentalement Thamnomys surdaster ou parasitant spontanément Galagoides demidovii (Fischer, 1808) sont identiques par leur morphologie céphalique, la longueur de leurs spicules et l'ornementation cuticulaire précloacale. Nous pensons qu'ils appartiennent à une même espèce.

Ces mâles diffèrent de $P$. soricina par certains caractères céphaliques et génitaux.

Leurs papilles submédianes sont en effet moins dilatées que celles de $P$. soricina et les trois dents de chaque pseudolèvre sont plus réduites. En outre, les lignes cuticulaires précloacales sont inexistantes chez $P$. soricina.

Il apparaît donc que l'espèce parasite de Galagoides demidovii diffère principalement de $P$. soricina Baylis, 1934, par des structures céphaliques moins différenciées, par une ornementation plus soutenue de la bourse caudale du mâle et par un comportement différent dans le tube digestif de l'hôte. Nous considérons qu'elle est distincte de $P$. soricina et qu'elle est nouvelle dans le genre Pseudophysaloptera.

Nous la nommons $P$. vincenti la dédiant à notre collègue F. Vincent de Brazzaville et identifions à cette espèce les larves du troisième stade infestant spontanément l'Insecte Labidura riparia.

(*) Les limites spécifiques des Pseudophysaloptères sont particulièrement difficiles à préciser car l'évolution se fait vers une atrophie des structures (réduction des spicules et des papilles caudales).

Baer (1959) et Schad (1963) ont identifié à $P$. soricina des spécimens de plus grande taille chez lesquels les spicules sont chitinoïdes et les papilles mieux individualisées.

Une étude expérimentale serait nécessaire pour chercher à connaître les variations morphologiques liées à l'âge, mais nos spécimens récoltés chez Crocidura sp. correspondent précisément à la description originale où aucune trace de spicules n'a été trouvée et peuvent être nommés sans difficulté. 


\section{Bibliographie}

BAER (J.-G.), 1959. - Helminthes parasites. Exploration des Parcs Nationaux du Congo Belge. Mission J.-G. Baer, W. Gerber. - Inst. Parc Nat. Congo Belge, 1, p. 1-163, fig. 1-94, pl. I-VIII.

Basir (M. A.), 1948. - On a Physaloptera larva from an Insect. Canad. Journ. Res. D. 26, p. 197-200, fig. 1-2.

Baylis (H. A.), 1934. - On a collection of Cestodes and Nematodes from small Mammals in Tanganyika Territory. - Ann. Mag. Nat. Hist., ser. 10, 13, p. 338-353, fig. 1-6.

Schad (G. A.), 1963. - A Taxonomic revision of the genus Pseudophysaloptera Baylis, 1934 (Nematoda : Physalopteridae). - Canad. Journ. Zool., 41, p. 1069-1077, fig. 1-11.

SkrJabin (K. I.) et Sobolev (A. A.), 1964. - Osnovi Nematodologii. - Acad. Sc. U.R.S.S., 12, p. $1-333$, fig. $1-195$. 